\title{
A GENERALIZATION OF ABSOLUTE RIESZIAN SUMMABILITY
}

B. J. BOYER AND L. I. HOLDER

1. Introduction. Absolute Rieszian summability was defined in 1928 by N. Obreschkoff $[4 ; 5]$ as follows:

Definition 1. Let $k>0$, and $0 \leqq \lambda_{1}<\lambda_{2}<\cdots<\lambda_{n}, \lambda_{n} \rightarrow \infty$ as $n \rightarrow \infty$. Let

$$
C_{\lambda}^{k}(\omega)=\sum_{\lambda_{n} \leqq \omega} a_{n}\left(1-\frac{\lambda_{n}}{\omega}\right)^{k}
$$

If the integral

$$
\int_{a}^{\infty}\left|\frac{d}{d \omega} C_{\lambda}^{k}(\omega)\right| d \omega<\infty, \quad a \geqq 0,
$$

then $\sum a_{n}$ is said to be absolutely summable by Rieszian means of order $k$ and type $\lambda$, or summable $|R, \lambda, k|$.

The case $\lambda_{n}=n$ is of particular interest in this paper. Summability $|R, n, k|$ has been shown by J. M. Hyslop [3] to be equivalent to absolute Cesàro summability of order $k$, or summability $|C, k|$. One of the principal results shown by Obreschkoff was the consistency of the $|R, n, k|$ means; that is, he showed that summability $|R, n, k|$ implies summability $\left|R, n, k^{\prime}\right|$, where $k^{\prime}>k$.

In this paper we introduce a method of absolute summability based upon the $(\alpha, \beta)$ method of summability defined by Bosanquet and Linfoot [1]. Just as the Bosanquet-Linfoot method generalized Riesz's arithmetic mean $(R, n, \alpha)$, the method given here will generalize absolute Rieszian summability $|R, n, \alpha|$.

Definition 2. A series $\sum a_{n}$ is said to be absolutely summable $(\alpha, \beta)$, or summable $|\alpha, \beta|$, where $\alpha>0$ or $\alpha=0, \beta>0$, if for each sufficiently large $C$,

$$
\int_{0}^{\infty}\left|\frac{d}{d \omega} A_{\alpha, \beta}(\omega)\right| d \omega<\infty,
$$

where

$$
A_{\alpha, \beta}(\omega)=\sum_{n<\omega} B\left(1-\frac{n}{\omega}\right)^{\alpha} \log ^{-\beta} \frac{C}{1-n / \omega} a_{n}
$$

Received by the editors April 3, 1962. 
and $B=\log ^{\beta} C$. Summability $|0,0|$ is defined to be absolute convergence.

Thus $|\alpha, 0|$ summability is the same as $|R, n, \alpha|$ summability. Condition (1) is equivalent to the bounded variation of $A_{\alpha, \beta}(\omega)$ in $(0, \infty)$. (See $[2$, p. 605].)

In the present paper it will be proved that $|\alpha, \beta|$ summability is consistent in the following sense: $|\alpha, \beta|$ summability implies $\left|\alpha^{\prime}, \beta^{\prime}\right|$ summability, where either $\alpha^{\prime}>\alpha$ or $\alpha^{\prime}=\alpha, \beta^{\prime}>\beta$. In a future paper, the authors propose to show some applications of $|\alpha, \beta|$ summability analogous to known results for absolute Rieszian, or Cesàro, summability.

\section{Lemmas.}

Lemma 1. Let $f(x), k(u)$, and $K(u)$ satisfy the following conditions:

(i) For some $n \geqq 0, V_{0}^{T}\left(x^{-n} f(x)\right)<\infty$ for all $T>0$. (It will be assumed throughout that for $x=0$, the function $x^{-n} f(x)$ is replaced by $\lim _{x \rightarrow+0} x^{-n} f(x)$.)

(ii) $k(u)$ is absolutely continuous in $[0,1]$.

(iii) $K(u)$ is positive, continuously differentiable in $[0,1)$, Lebesgue integrable over $[0,1], \lim _{u \rightarrow 1^{-}} K(u)=+\infty$ and $u K^{\prime}(u) / K(u)$ is nondecreasing.

Let

$$
F(x)=x^{-n} \int_{0}^{1} K(u) f(x u) d u ; \quad G(x)=x^{-n} \int_{0}^{1} k(u) K(u) f(x u) d u .
$$

Then $V_{0}^{\infty} G(x) \leqq \gamma V_{0}^{\infty} F(x)$, where $\gamma=\int_{0}^{1}\left|k^{\prime}(u)\right| d u+|k(1)|$.

Proof. For $T>0$ let $p$ be a partition, $0=x_{0}<x_{1}<\cdots<x_{N}=T$, of $[0, T]$. Corresponding to this partition let us define

$$
\Delta\left(f_{i}, u\right)=x_{i}^{-n} f\left(u x_{i}\right)-x_{i-1}^{-n} f\left(u x_{i-1}\right)
$$

and

$$
\Delta G_{i}=G\left(x_{i}\right)-G\left(x_{i-1}\right), \quad i=1,2, \cdots, N .
$$

Then

$$
\sum_{(p)}\left|\Delta G_{i}\right|=\sum_{(p)}\left|\int_{0}^{1} k(u) K(u) \Delta\left(f_{i}, u\right) d u\right| .
$$

An integration by parts of the right side of (3) leads to the inequality

$$
\sum_{(p)}\left|\Delta G_{i}\right| \leqq C_{1}+\int_{0}^{1}\left|k^{\prime}(u)\right| \sum_{(p)}\left|\int_{0}^{u} K(t) \Delta\left(f_{i}, t\right) d t\right| d u
$$


where $C_{1}=|k(1)| V_{0}^{T} F(x)$. Since $\sum_{(p)}\left|\int_{0}^{u} K(t) \Delta\left(f_{i}, t\right) d t\right|$ is a continuous function of $u$, (4) becomes, with the aid of the first mean-value theorem,

$$
\sum_{(p)}\left|\Delta G_{i}\right| \leqq C_{1}+C_{2} \sum_{(p)}\left|\int_{0}^{u_{0}} K(u) \Delta\left(f_{i}, u\right) d u\right|,
$$

where $C_{2}=\int_{0}^{1}\left|k^{\prime}(u)\right| d u$ and $0 \leqq u_{0} \leqq 1$.

If $u_{0}=0$ or 1 , the right side of (5) is clearly no greater than $\gamma V_{0}^{T} F(x)$, where $\gamma=\int_{0}^{1}\left|k^{\prime}(u)\right| d u+|k(1)|$. If $0<u_{0}<1$, then after changing variables and integrating by parts, (5) becomes

$$
\begin{aligned}
\sum_{(p)}\left|\Delta G_{i}\right| \leqq C_{1} & +C_{2}\left\{\sum_{(p)} \mid \frac{u_{0} K\left(u u_{0}\right)}{K(u)} \int_{0}^{u} K(t) \Delta\left(f_{i}, t u_{0}\right) d t\right]_{u=0}^{u=1} \\
& \left.-u_{0} \int_{0}^{1} \frac{d}{d u}\left(\frac{K\left(u u_{0}\right)}{K(u)}\right) \int_{0}^{u} K(t) \Delta\left(f_{i}, t u_{0}\right) d t d u \mid\right\} .
\end{aligned}
$$

But hypothesis (iii) implies that the integrated part vanishes at both limits, and that $(d / d u)\left\{K\left(u u_{0}\right) / K(u)\right\} \leqq 0$. Again applying the first mean-value theorem, it follows from (6) that

$$
\sum_{(p)}\left|\Delta G_{i}\right| \leqq C_{1}+C_{2} u_{0} \sum_{(p)}\left|\int_{0}^{u_{1}} K(u) \Delta\left(f_{i}, u u_{0}\right) d u\right|,
$$

where $0 \leqq u_{1} \leqq 1$.

Repetition of the steps leading from (5) to (7) gives the result,

$$
\sum_{(p)}\left|\Delta G_{i}\right| \leqq C_{1}+C_{2} \Pi_{m} \sum_{(p)}\left|\int_{0}^{1} K\left(u u_{m}\right) \Delta\left(f_{i}, u \Pi_{m}\right) d u\right|,
$$

where

$$
\Pi_{m}=\prod_{\nu=0}^{m} u_{\nu}, \quad 0 \leqq u_{\nu} \leqq 1, \quad u_{\nu} \neq 0,1 \text { for } \nu<m, \quad m=1,2, \cdots
$$

From (8) we shall deduce that

$$
\sum_{(p)}\left|\Delta G_{i}\right| \leqq \gamma V_{0}^{T} F(x)
$$

There are two cases to consider.

Case 1. For some $m$, either $u_{m}=0$ or 1 . It is not difficult to verify then that $\sum_{(p)}\left|\Delta G_{i}\right| \leqq C_{1}$, or $\sum_{(p)}\left|\Delta G_{i}\right| \leqq C_{1}+C_{2}\left(\Pi_{m}\right)^{n+1} V_{0}^{T \Pi_{m}} F(x)$, respectively. In either case (9) is clearly satisfied. This case for $m=0$ has been settled already. 
Case 2. Suppose $u_{m} \neq 0,1$ for all $m$. Since $\left\{\Pi_{m}\right\}$ is a monotone sequence, $\Pi_{m} \rightarrow L$ as $m \rightarrow \infty, 0 \leqq L<1$. If $L=0$ then

$$
\Pi_{m} \sum_{(p)}\left|\int_{0}^{1} K\left(u u_{m}\right) \Delta\left(f_{i}, u \Pi_{m}\right) d u\right| \leqq 2 M N\left(\Pi_{m}\right)^{n+1} \int_{0}^{1} K(u) d u=o(1)
$$

as $m \rightarrow \infty$, where $M=1$.u.b. $\left[x^{-n} f(x)\right]$ over $[0, T]$. Hence (9) holds when $L=0$.

Finally, if $L \neq 0$, then necessarily $\lim _{m \rightarrow \infty} u_{m}=1$. Since each integrand in (8) is majorized by a summable function, a well-known theorem of Lebesgue integration may be applied to (8) to give

$$
\begin{aligned}
\sum_{(p)}\left|\Delta G_{i}\right| & \leqq C_{1}+C_{2} L \sum_{(p)}\left|\int_{0}^{1} K(u) \Delta\left(f_{i}, u L\right) d u\right| \\
& \leqq C_{1}+C_{2} L^{n+1} V_{0}^{T L} F(x) \\
& \leqq \gamma V_{0}^{T} F(x) .
\end{aligned}
$$

Thus the truth of (9) has been established for each partition $p$ and each $T>0$. From (9) it follows that $V_{0}^{T} G(x) \leqq \gamma V_{0}^{T} F(x)$, and from this the lemma.

Lемма 2. Lemma 1 remains valid if condition (iii) is replaced by: (iii)*. $K(u)$ is constant in $[0,1]$.

Proof. An argument similar to that in the preceding lemma will show that (8) also holds under (iii)*. Then (9) is easily verified, and the conclusion follows.

\section{The consistency theorem.}

Theorem. If $\sum a_{n}$ is summable $|\alpha, \beta|$, then it is summable $\left|\alpha^{\prime}, \beta^{\prime}\right|$, for $\alpha^{\prime}>\alpha$, or $\alpha^{\prime}=\alpha, \beta^{\prime}>\beta$.

Proof.

Case 1. $\alpha=\beta=0$. We must show that absolute convergence of the series implies $\left|\alpha^{\prime}, \beta^{\prime}\right|$ summability, where $\alpha^{\prime}>0$ or $\alpha^{\prime}=0, \beta^{\prime}>0$. Let

$$
\begin{aligned}
& \Phi_{\alpha, \beta}(u)=B u^{\alpha} \log ^{-\beta} \frac{C}{u}, \quad \text { if } u \neq 0, \\
& \Phi_{\alpha, \beta}(0)=0, \quad \text { if } \alpha>0 \text { or } \alpha=0, \beta>0 .
\end{aligned}
$$

Then, what we have to show is the convergence of the integral

$$
\int_{0}^{\infty}\left|\frac{1}{\omega^{2}} \sum_{n<\omega} \Phi_{\alpha^{\prime}, \beta^{\prime}}^{\prime}\left(1-\frac{n}{\omega}\right) n a_{n}\right| d \omega
$$


Noting that for $n<\omega, \Phi_{\alpha^{\prime}, \beta^{\prime}}^{\prime}(1-n / \omega)>0$ for sufficiently large $C$, we have ${ }^{1}$

$$
\begin{aligned}
\int_{0}^{\infty} & \left|\frac{1}{\omega^{2}} \sum_{n<\omega} \Phi_{\alpha^{\prime}, \beta^{\prime}}^{\prime}\left(1-\frac{n}{\omega}\right) n a_{n}\right| d \omega \\
& \leqq \int_{0}^{\infty} \sum_{n<\omega}\left|a_{n}\right| \frac{n}{\omega^{2}} \Phi_{\alpha^{\prime}, \beta^{\prime}}^{\prime}\left(1-\frac{n}{\omega}\right) d \omega \\
& \leqq \sum_{n=0}^{\infty}\left|a_{n}\right| \int_{n}^{\infty} \frac{n}{\omega^{2}} \Phi_{\alpha^{\prime}, \beta^{\prime}}^{\prime}\left(1-\frac{n}{\omega}\right) d \omega \\
& =\sum_{n=0}^{\infty}\left|a_{n}\right| \int_{0}^{1} \Phi_{\alpha^{\prime}, \beta^{\prime}}^{\prime}(u) d u \\
& =\sum_{n=0}^{\infty}\left|a_{n}\right| .
\end{aligned}
$$

The result now follows, since $\sum\left|a_{n}\right|$ is finite.

Case 2. $\alpha>0$, or $\alpha=0, \beta>0$. In this case it is known $[1$, p. 209] that $A_{\alpha, \beta}(\omega)$ has the integral representation,

$$
A_{\alpha, \beta}(\omega)=\int_{0}^{1} \Phi_{\alpha, \beta}^{\prime}(1-u) A(\omega u) d u,
$$

where $A(x)=\sum_{n \leq x} a_{n}$. Let $h=[\alpha]$; then as in $\left[1\right.$, p. 216] $A_{\alpha, \beta}(\omega)$ may be written in the following forms:

$$
A_{\alpha, \beta}(\omega)=\omega^{-j} \int_{0}^{1} \Phi_{\alpha, \beta}^{(j+1)}(1-u) A_{j}(\omega u) d u,
$$

for $j=0,1, \cdots, h$, if $\alpha=h, \beta>0$ or $h<\alpha<h+1$; for $j=0,1, \cdots$, $h-1$, if $\alpha=h \geqq 1, \beta \leqq 0$; where $A_{j}(x)=\int_{0}^{x} A_{j-1}(t) d t$ and $A_{0}(x)=A(x)$.

By choosing the appropriate form in (11), one finds that for $\alpha^{\prime}>\alpha$ or $\alpha^{\prime}=\alpha, \beta^{\prime}>\beta$,

$$
A_{\alpha^{\prime}, \beta^{\prime}}(\omega)=\omega^{-(h-1)} \int_{0}^{1} \frac{\Phi_{\alpha^{\prime}, \beta^{\prime}}^{(h)}(1-u)}{\Phi_{\alpha, \beta}^{(h)}(1-u)} \Phi_{\alpha, \beta}^{(h)}(1-u) A_{h-1}(\omega u) d u
$$

when $\alpha=h, \beta \leqq 0$, and

$$
A_{\alpha^{\prime}, \beta^{\prime}}(\omega)=\omega^{-h} \int_{0}^{1} \frac{\Phi_{\alpha^{\prime}, \beta^{\prime}}^{(h+1)}(1-u)}{\Phi_{\alpha, \beta}^{(h+1)}(1-u)} \Phi_{\alpha, \beta}^{(h+1)}(1-u) A_{h}(\omega u) d u
$$

when $\alpha=h, \beta>0$ or $h<\alpha<h+1$.

${ }^{1}$ For justification of interchange of order of summation and integration, see, e.g., Titchmarsh [6, p. 348]. 
A routine calculation shows that the first and second factors of the integrands (12) and (13) satisfy the requirements for $k(u)$ and $K(u)$, respectively, in Lemma 1 or 2 (whichever is applicable) for $C$ sufficiently large. The theorem now follows immediately from these two lemmas.

\section{REFERENCES}

1. L. S. Bosanquet and E. H. Linfoot, Generalized means and the summability of Fourier series, Quart. J. Math. Oxford Ser. (2) 2 (1931), 207-229.

2. E. W. Hobson, The theory of functions of a real variable. I, 3rd. ed., Harren Press, Washington, D. C., 1950.

3. J. M. Hyslop, On the absolute summability of series by Rieszian means, Proc. Edinburgh Math. Soc. (2) 5 (1936), 46-54.

4. N. Obreschkoff, Sur la sommation absolue des series de Dirichlet, C.R. Acad. Sci. Paris 186 (1928), 215-217.

5. - Über die absolute Summierung der Dirichletschen Reihen, Math. Z. 30 (1929), 375-386.

6. E. C. Titchmarsh, The theory of functions, 2nd ed., Oxford Univ. Press, London, 1939.

Florida State University and

San Jose State College 